7th International Workshop on Astronomy and

Relativistic Astrophysics (IWARA 2016)

International Journal of Modern Physics: Conference Series

Vol. 45 (2017) 1760003 (9 pages)

(C) The Author(s)

DOI: $10.1142 / \mathrm{S} 2010194517600035$

\title{
Conformal Gravity Solutions to Standard Gravity Problems
}

\author{
James G. O'Brien \\ Department of Sciences, Wentworth Institute of Technology \\ 550 Huntington Ave., Boston, MA 02115, USA \\ obrienj10@wit.edu \\ Spasen Chaykov \\ Department of Physics, Brandeis University \\ 415 South St., Waltham, MA 02451, USA \\ chaykovs@brandeis.edu \\ Thomas L. Chiarelli \\ Department of Electromechanical Engineering, \\ Wentworth Institute of Technology \\ 550 Huntington Ave, Boston, MA 02115, USA \\ chiarellit@wit.edu \\ Taylor Saintable, Justin Harrington \\ Department of Applied Mathematics, \\ Computer Engineering, Wentworth Institute of Technology \\ 550 Huntington Ave, Boston, MA 02115, USA \\ saintablet@wit.edu,harringtonj5@wit.edu
}

Published 15 August 2017

\begin{abstract}
Gravitational theories outside standard general relativity have been drawing increased attention over the past several years, mostly due to the lack of direct observational evidence of dark matter. With some recent very high level dark matter searches continuing, and the parameter space to search decreasing has lead to a new interest in rethinking gravity at the largest of scales. As an alternative gravitational theory, conformal gravity has enjoyed much of the success of Modified Newtonian Dynamics (MOND) in predicting phenomenology, but differs dramatically in its initial construction. In this work, we explore some recent advances in conformal gravity, which help to build the case for support of such an alternative theory. Here, we highlight conformal gravity's success in
\end{abstract}

This is an Open Access article published by World Scientific Publishing Company. It is distributed under the terms of the Creative Commons Attribution 4.0 (CC-BY) License. Further distribution of this work is permitted, provided the original work is properly cited. 
J. G. O'Brien et al.

fitting new rotation curves, its ability to explain velocity dispersions in clusters, the initial steps towards gravitational lensing and finally, some preliminary work on explaining universal centripetal acceleration trends in galaxies.

Keywords: Conformal Gravity; General Relativity; Dark Matter.

PACS numbers: 95.30.Sf, 03.30.+p, 95.35.+d.

\section{Introduction}

Conformal gravity is a fourth order renormalizable metric theory of gravity, where the Ricci scalar in the Einstein-Hilbert action is replaced by the square of the conformal Weyl tensor as:

$$
\begin{aligned}
I_{\mathrm{W}} & =-\alpha_{g} \int d^{4} x(-g)^{1 / 2} C_{\lambda \mu \nu \kappa} C^{\lambda \mu \nu \kappa} \\
& =-2 \alpha_{g} \int d^{4} x(-g)^{1 / 2}\left[R_{\mu \kappa} R^{\mu \kappa}-(1 / 3)\left(R_{\alpha}^{\alpha}\right)^{2}\right]
\end{aligned}
$$

where

$$
\begin{aligned}
C_{\lambda \mu \nu \kappa}= & R_{\lambda \mu \nu \kappa}-\frac{1}{2}\left(g_{\lambda \nu} R_{\mu \kappa}-g_{\lambda \kappa} R_{\mu \nu}-g_{\mu \nu} R_{\lambda \kappa}+g_{\mu \kappa} R_{\lambda \nu}\right) \\
& +\frac{1}{6} R^{\alpha}{ }_{\alpha}\left(g_{\lambda \nu} g_{\mu \kappa}-g_{\lambda \kappa} g_{\mu \nu}\right)
\end{aligned}
$$

is the conformal Weyl tensor and the gravitational coupling constant $\alpha_{g}$ is dimensionless. This action, when varied with respect to the metric (as in standard gravity $^{1}$ ), yields the conformal gravity field equations,

$$
4 \alpha_{g} W^{\mu \nu}=4 \alpha_{g}\left[2 C_{; \lambda ; \kappa}^{\mu \lambda \nu \kappa}-C^{\mu \lambda \nu \kappa} R_{\lambda \kappa}\right]=T^{\mu \nu} .
$$

These equations were later solved by Mannheim and Kazanas ${ }^{2}$ for a spherical mass, exterior solution, which behaves as a conformal gravity Schwarzchild like solution, and allows for application of the theory to various data sets. In recent years, Mannheim and O'Brien have shown that conformal gravity can be used to accurately describe the rotation curves of a diverse set of galaxies without the need to invoke dark matter. ${ }^{3-6}$ Since all attempts to find any direct evidence of dark matter have been unsuccessful so far, alternative theories of gravity have become increasingly worth considering as an explanation for phenomena ascribed to dark matter. Its success in fitting galactic rotations makes conformal gravity an ideal candidate to explore in an effort to theoretically describe these additional effects. While the rotation curve fitting provides direct data that can be tested against the predictions of an alternative model of gravity, there are other observational motivations for the existence of dark matter, such as the motion of galaxies in clusters and gravitational lensing. The goal of this paper is to summarize some recent conformal gravity fits to rotation ${ }^{3-6}$ curves, to show how conformal gravity can address the cluster motion problem without invoking dark matter, and develop the initial framework for gravitational lensing formalism in conformal gravity. Since the actual IWARA 
meeting, recent work was published in Physical Review Letters ${ }^{7}(\mathrm{PRL})$, which generalizes rotation curve data to instead encapsulate what appears to be universality in the centripetal accelerations of the rotation curve data. Since the conference, the authors above have made an initial pass at conformal gravity's answer to this new found phenomena, and we report the preliminary findings in this work.

\section{Rotation Curves of the WHISP Survey}

In this paper, we review our recent efforts in fitting an extended, late-type dwarf galaxy survey, comprising of data from 62 galaxies taken at the Westerbork HI Survey of Spiral and Irregular Galaxies (WHISP ${ }^{8}$ ).

One of the authors has published the first 26 galaxies $^{5}$ in this survey, and now the remaining 36 conformal gravity fits have been produced. In Fig. 1, we present the General Relativity, conformal gravity and MOND rotation curve predictions for a selection of the entire set (MOND is out of the scope of this paper, but included in the figures for comparison purposes). This selection was chosen to represent the diverse distances, overall masses and luminosities represented in the full survey, and was consolidated to those shown simply for brevity. The General Relativity prediction $^{9}$ is given by,

$$
v_{g r}(R)=\sqrt{\frac{N^{*} \beta^{*} c^{2} R^{2}}{2 R_{0}^{3}}\left[I_{0}\left(\frac{R}{2 R_{0}}\right) K_{0}\left(\frac{R}{2 R_{0}}\right)-I_{1}\left(\frac{R}{2 R_{0}}\right) K_{1}\left(\frac{R}{2 R_{0}}\right)\right]},
$$

the conformal gravity prediction ${ }^{4}$ is given as,

$$
v_{c g}(R)=\sqrt{v_{g r}^{2}+\frac{N^{*} \gamma^{*} c^{2} R^{2}}{2 R_{0}} I_{1}\left(\frac{R}{2 R_{0}}\right) K_{1}\left(\frac{R}{2 R_{0}}\right)+\frac{\gamma_{0} c^{2} R}{2}-\kappa c^{2} R,}
$$

and the MOND prediction ${ }^{10}$ is given as,

$$
v_{\text {mond }}(R)=\sqrt{v_{g r}^{2}(R)+\left(v_{g r}^{2}(R) \frac{\sqrt{1+\frac{4 a_{0} R}{v_{g r}^{2}}-1}}{2}\right)} .
$$

In each of the above formula, $N^{*}$ is the estimated number of stars in the galaxy (proportional to the mass) and $R_{0}$ is the galactic scale length. In eq. (5), $\gamma^{*}=$ $5.42 * 10^{-41} \mathrm{~cm}^{-1}, \gamma_{0}=3.06 * 10^{-30} \mathrm{~cm}^{-1}$, and $\kappa=9.54 * 10^{-54} \mathrm{~cm}^{-2}$. In eq. (6), $a_{0}=1.35 \times 10^{-8} \mathrm{~cm} \mathrm{~s}^{-2}$ and we refer the reader to the original sources ${ }^{4,10}$ for the determination of these constants. It should be noted that in each of these models, both the luminous disk and gas were fit. The individual galaxies' total gas mass is given in Table 1 (where all quantities with the $\odot$ are in solar quantities). No bulges were included in any of these fits due to lack of observational ${ }^{8}$ evidence.

It can be easily seen in Fig. 1, that conformal gravity captures the essence of the data without the need for dark matter while generating mass models that return mass to light ratios consistent with physical observations (see Table 1). Further, in 

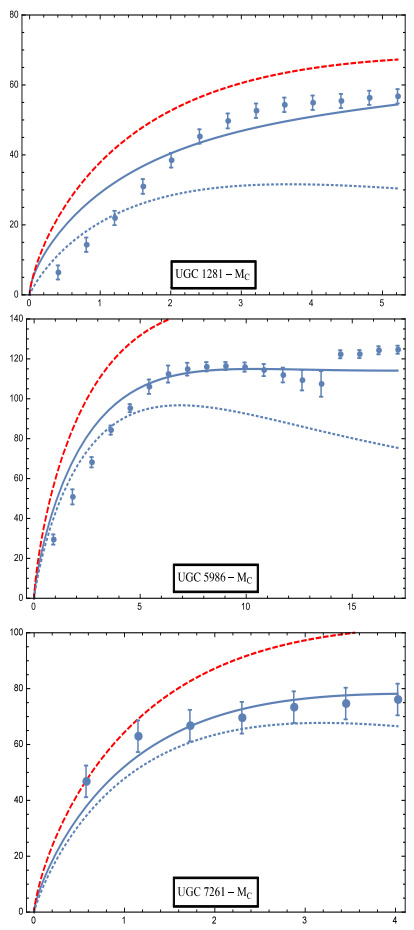
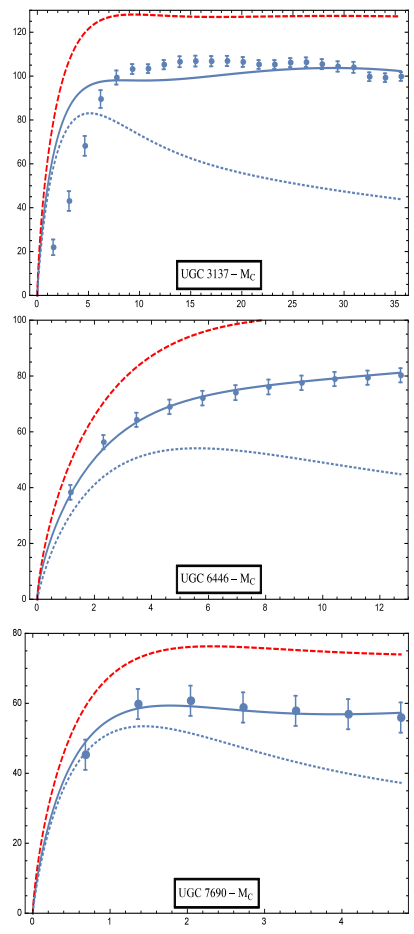
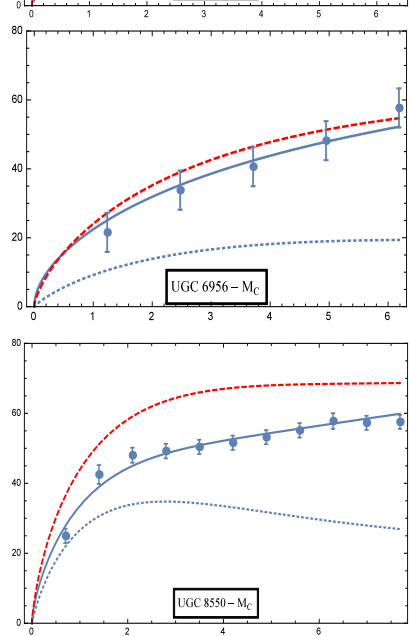

Fig. 1. Fitting to the rotational velocities $(\mathrm{km} / \mathrm{s})$ of the WHISP sample with quoted errors as a function of radial distance $(\mathrm{kpc})$. Blue dashed curve $=$ Newtonian, Full blue curve $=$ conformal gravity, Full red curve $=$ MOND. No dark matter is assumed.

the last column of Table 1, we see the same trend that universality can be found in the data, where the number $\left(\frac{v^{2}}{c^{2} R_{l}}\right)_{\text {last }}$ seems to be of order one, for now over 200 galaxies $^{3-6}$ fit by the theory. This universality found in the last data point of each galaxies centripetal acceleration (scaled here to $\frac{1}{c^{2}}$ ) provides argument for the recent work of $\mathrm{McGaugh}^{7}$ et al., and this phenomena will be explored in more detail below.

\section{Conformal Gravity and Cluster Motion}

While the dominant portion of the literature on alternative gravitational models focuses on the rotation curve ${ }^{11,12}$ problem, there are plenty of other astronomical observations which point to the existence of dark matter. The original motivator, was Zwicky's 1930's work which has a mass discrepancy when comparing luminous observations of galactic cluster motions, to the gravitational predictions. As time went on, the discrepancy he noted only proved to be more substantial than initially thought. Using the luminous data to extract velocities of the galaxies, he was able to make mass estimates of the Coma and Virgo clusters. One can arrive 
Table 1. Properties of the galaxy sample.

\begin{tabular}{cccccccc}
\hline \hline $\begin{array}{c}\text { Galaxy } \\
\text { UGC }\end{array}$ & $\begin{array}{c}\text { Dist. } \\
(\mathrm{Mpc})\end{array}$ & $\begin{array}{c}\text { Lum. } \\
\left(10^{8} L \odot\right)\end{array}$ & $\begin{array}{c}R_{0} \\
(\mathrm{kpc})\end{array}$ & $\begin{array}{c}M_{\text {gas }} \\
\left(10^{8} M_{\odot}\right)\end{array}$ & $\begin{array}{c}M_{d} \\
\left(10^{8} M_{\odot}\right)\end{array}$ & $\begin{array}{c}(M / L) \\
\left(M_{\odot} L_{\odot}^{-1}\right)\end{array}$ & $\begin{array}{c}\left(v^{2} / c^{2} R_{l}\right)_{\text {last }} \\
\left(10^{-30} \mathrm{~cm}^{-1}\right)\end{array}$ \\
\hline 1281 & 5.5 & 4.7 & 1.66 & 3.2 & 9.54 & 2.02 & 2.22 \\
3137 & 21.2 & 62.7 & 2.25 & 58.1 & 86.30 & 1.38 & 1.01 \\
5829 & 8.0 & 10.2 & 1.72 & 8.8 & 10.16 & 0.99 & 2.65 \\
5986 & 12.4 & 87.1 & 3.11 & 55.1 & 166.99 & 1.92 & 3.26 \\
6446 & 15.9 & 57.1 & 2.48 & 23.7 & 40.65 & 0.71 & 1.82 \\
6956 & 17.0 & 13.8 & 2.57 & 9.6 & 4.69 & 0.34 & 1.94 \\
7261 & 7.9 & 14.1 & 1.47 & 5.1 & 39.41 & 2.80 & 5.18 \\
7690 & 9.4 & 13.8 & 0.64 & 5.2 & 10.31 & 0.75 & 2.37 \\
8550 & 9.6 & 8.9 & 1.21 & 5.9 & 8.12 & 0.91 & 1.55 \\
\hline \hline
\end{tabular}

Table 1 Entries from left to right are: Galaxy name, NED Distance, Total Blue Luminosity, Disk Scale Length, Distance of the last data point, Mass of the gas (including helium), disk mass, mass to light ratios, scaled centripetal acceleration of the last observed data point.

at the mass estimates via the virial theorem, upon using the measured velocity dispersion $\sigma$ to get the average velocity ${ }^{13}\left\langle v^{2}\right\rangle \approx 3 \sigma^{2}$. It is important to note that this original Zwicky method will ignore systematic effects such as rotational or tangental motions. However, this calculation is enough to provide the order of magnitudes in question. For a complete treatment of how the individual corrections should be implemented, see Mannheim ${ }^{14}$ 2006. For the Coma cluster, the current measured distance and velocity dispersions are $\sigma_{\text {Coma }}^{2}=3.413 * 10^{12} \frac{\mathrm{m}^{2}}{\mathrm{~s}^{2}}$ and $R_{\text {Coma }}=1.206 \mathrm{Mpc}$, with an estimated ${ }^{15} 1000$ galaxies. Hence, we arrive at the mass of a galaxy in the Coma Cluster is $9.5 * 10^{11} M_{\odot}$. It is important to note that this mass places all galaxies into the common morphology of huge spirals. From this, one obtains the mass to light ratio $\gamma=\frac{M}{L} \approx 200 \gamma_{\odot}$. Since physical mass to light ratios should be about the order of unity, ${ }^{9}$ this shows the initial missing mass problem. Conformal gravity can mirror this calculation for the mass predictions in the cluster. Mannheim and Kazanas, solved the fourth order exterior metric ${ }^{16}$ to a spherical source, from which we extract the weak field potential,

$$
V=-\frac{G M}{r}+\frac{M \gamma^{*} c^{2} r}{2 M_{\odot}}+\frac{\gamma_{0} c^{2} r}{2}-k c^{2} r^{2} .
$$

Applying the same logic as Zwicky, the conformal gravity mass estimate is given by

$$
M \approx \frac{\sigma^{2}-\frac{\gamma_{0} c^{2} R}{2}+k c^{2} R^{2}}{\frac{G}{R}+\frac{\gamma^{*} c^{2} R}{2 M_{\odot}}},
$$

which for $\mathrm{R}$ on the scale of the virial radius of a cluster can be simplified to $M \approx$ $\frac{2 k}{\gamma^{*}} R M_{\odot}$. Using the same values as above, the conformal estimate for an average galaxy in the Coma cluster is $1.3 * 10^{9} M_{\odot}$. This value adheres to a more diverse set of morphologies across the Coma cluster. ${ }^{17}$ The corresponding mass to light ratio of $\gamma=\frac{M}{L} \approx 0.26 \gamma_{\odot}$ shows that the conformal theory does not yield a missing mass problem. Although this is a singular cluster amongst many, we can repeat the procedure above for the Virgo cluster $^{15}$ to yield the mass to light ratio of 


\section{J. G. O'Brien et al.}

$\gamma=\frac{M}{L} \approx 1.02 \gamma_{\odot}$. Further work is being done on the SPT-SZ ${ }^{18}$ survey, to extend the amount of cluster information modeled by conformal gravity.

\section{Gravitational Bending of Light in Conformal Gravity}

The 1919 experiment which verified the gravitational bending of light was one of the great triumphs of general relativity. Since this prediction, the bending of light has been used for explanation of large scale phenomena through the idea of gravitational lensing, typically caused by the presence of dark matter. Since conformal gravity acts to replace dark matter with new large scale physics, as an alternative theory of gravity, it is required of the theory to have an explanation for the lensing phenomena. In order to develop the full conformal gravity prediction about lensing (out of the scope of this review), we must first establish the conformal gravity prediction of the deflection of starlight. At the local level, we can ignore the global quadratic ${ }^{2}$ term, and return to the metric of a spherical source ${ }^{16}$ in conformal gravity,

$$
d s^{2}=-\left(1-\frac{2 G M}{c^{2} r}+\frac{M \gamma^{*} r}{M_{\odot}}\right) c^{2} d t^{2}+\frac{1}{\left(1-\frac{2 G M}{c^{2} r}+\frac{M \gamma^{*} r}{M \odot}\right)} d r^{2}+r^{2} d \theta^{2}+r^{2} \sin ^{2} \theta d \phi^{2} .
$$

Taking this metric, for the simple case of two dimensional deflection, with the sun as the gravitational source, we expand the metric to become,

$$
d s^{2}=-\left(1-\frac{2 G M}{c^{2} r}+\frac{M \gamma^{*} r}{M_{\odot}}\right) c^{2} d t^{2}+\left(1+\frac{2 G M}{c^{2} r}-\frac{M}{M_{\odot}} \gamma^{*} r\right) d r^{2}+r^{2} d \theta^{2},
$$

where $\phi=0$. Orienting the system such that $\theta$ is the deflection angle, ${ }^{19}$ we can apply Huyghens Principle, to yield

$$
d s^{2}=-\left(1-\frac{2 G M}{c^{2} r}+\frac{M}{M_{\odot}} \gamma^{*} r\right) c^{2} d t^{2}+\left(1+\frac{2 G M x^{2}}{c^{2} r^{3}}-\frac{M \gamma^{*} x^{2}}{M_{\odot} r}\right) d x^{2} .
$$

Assuming light follows the null geodesics, this yields the coordinate velocity of the light $v$, as

$$
v=\frac{d x}{d t}=\left[1+\left(\frac{M \gamma^{*} r}{2 M_{\odot}}-\frac{G M}{c^{2} r}\right)\left(1+\frac{x^{2}}{r^{2}}\right)\right] c .
$$

Converting the $\mathrm{x}$ coordinate to polar coordinates, this expression can be integrated to yield the total deflection angle,

$$
\theta \approx \frac{4 G M}{c^{2} r_{0}}+\frac{M \gamma^{*} r_{0}}{2 M_{\odot}}
$$

The full calculation of these integrations are carried out in $\mathrm{O}^{\prime} \mathrm{Brien}^{20}$ et al. We can see in 13 the original Einstein prediction with the conformal gravity correction. Over larger distances we would also expect to start noticing the global effects of curvature and thus it would be instructive to derive the conformal bending of light by following the same procedure described above, but with the inclusion of the quadratic term. This would then allow for the discussion of predictions of weak lensing. This calculation is a first step towards this end, and work is currently underway to finalize the prediction. 


\section{Universal Centripetal Accelerations in Spiral Galaxies}

Recently, a secondary approach to rotation curve physics is under investigation based upon a correlation between the radial acceleration ${ }^{7}$ observed and that which is predicted by the distribution of baryonic matter. In this work, McGaugh et al. is able to constrain the amount of dark matter needed in the rotation curve paradigm and find a function ${ }^{7}$ which can explain the data. Another possible conclusion of this paper is that perhaps new physics is needed to explain this phenomena. Since conformal gravity seeks to dispense the need for dark matter, we reproduce the McGaugh fit in Fig. 2(a), and create a fit showing conformal gravities expectation in Fig. 2(b). It should be noted that the figure reproduced in Fig. 2(a) is slightly different due to the fact that we did not use the exact same galaxy set as in $\mathrm{McGaugh}^{7}$ et al. However, over 60 galaxies and 2000 unfiltered data points of varying galactic characteristics were analyzed and plotted in Fig. 2, capturing the original fit of McGaugh. If the laws of physics are universal, any data points plotted in terms of observed vs predicted must fall on the line of unity. In Fig. 2(b), we examine the predicted radial acceleration of General Relativity (blue) and conformal gravity (orange). The data predicted by GR hits the line of unity at accelerations greater than $10^{-9.5} \mathrm{~ms}^{-2}$ implying no need for dark matter at this scale, but then trails off for smaller accelerations. Conformal gravity however, accurately predicts the radial acceleration universally, for all acceleration values regardless of the varying galactic differences. Nearly all $2000+$ data points are within much less than one order of magnitude of the line of unity and no dark matter was needed, showing that conformal gravity has captured all aspects of the rotation curve problem. A more robust discussion of this feature of conformal gravity is in preparation for publication.

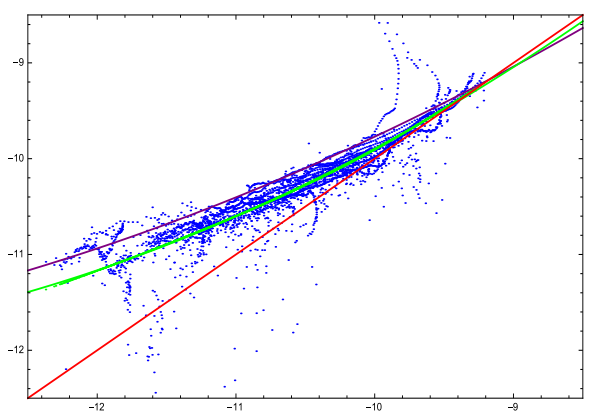

(a)

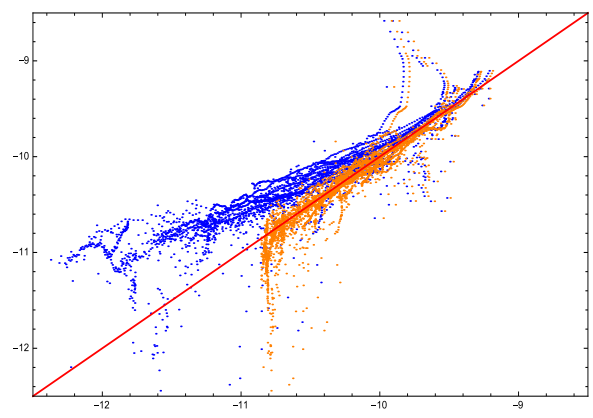

(b)

Fig. 2. Log-log plots of the observed radial accelerations $\left(g_{o b s}\right)$ vs. the predicted baryonic radial accelerations $\left(g_{b a r}\right)$. In Fig. 2(a) and Fig. 2(b) the baryonic acceleration as predicted by GR alone $\left(g_{b a r}\right)$ is in blue, and the line of unity is in red. Fig.2(a) is a reproduction of McGaugh ${ }^{7}$ et. al. using our data. The purple fit is taken directly from McGaugh ${ }^{7}$ et. al. and the green fit is the same function but renormalized to our data set. Fig 2(b) shows the prediction through incorporating conformal gravity as the prediction for $\left(g_{b a r}\right)$, and is shown in orange, overlaid with that of standard gravity (blue). 


\section{Conclusion}

In this paper we reviewed some recent important advancements in conformal gravity. Once again, conformal gravity provides an accurate description to newly obtained rotation curves, increasing the net total of rotation curves fit by the theory to over 200 galaxies. Second, we show that conformal gravity is equipped to solve the original missing mass problem as found by Zwicky, since it can model cluster dynamics without needing any additional mass. Third, we show the first initial steps towards applying conformal gravity to gravitational lensing, with the reproduction of the gravitational deflection of starlight calculation, which serves as a primer for a more robust lensing discussion. Lastly, we have shown how conformal gravity can explain the universal centripetal accelerations present in spiral galaxies on a 1:1 basis without the need for dark matter.

\section{Acknowledgments}

The Authors would like to thank the the organizers of the 7th International Workshop on Astronomy and Relativistic Astrophysics, as well as the hospitality of the city of Gramado Brazil for a wonderful conference. J. G. O'Brien would also like to thank Philip Mannheim for his continued guidance in advancing conformal gravity.

\section{References}

1. S. Weinberg, Gravitation and Cosmology: Principles and Applications of the General Theory of Relativity (New York,Wiley, 1972).

2. P. D. Mannheim, Astro - ph 9504022 (1995).

3. P. D. Mannheim and J. G. O'Brien, Phys. Rev. Lett. 106, p. 121101 (Mar 2011).

4. P. D. Mannheim and J. G. O'Brien, Physical Review D 85, p. 124020 (Jun 2012).

5. J. G. OBrien and P. D. Mannheim, Monthly Notices of the Royal Astronomical Society 421, 1273 (2012).

6. J. G. O'Brien and R. J. Moss, Journal of Physics: Conference Series 615, p. 012002 (2015).

7. S. McGaugh, F. Lelli and J. Schombert, ArXiv e-prints (September 2016).

8. R. A. Swaters, R. Sancisi, T. S. van Albada and J. M. van der Hulst, aap 493, 871 (January 2009).

9. P. D. Mannheim, Progress in Particle and Nuclear Physics 56, 340 (April 2006).

10. B. Famaey and S. S. McGaugh, Living Reviews in Relativity 15, p. 10 (September 2012).

11. S. Cisneros, N. S. Oblath, J. A. Formaggio, R. A. Ott, D. Chester, D. J. Battaglia, A. Ashley, R. Robinson and A. Rodriguez, ArXiv e-prints (July 2014).

12. J. R. Brownstein and J. W. Moffat, A. J. 636 (2006).

13. O. Knill, Springer (2007).

14. P. D. Mannheim, Linear Potentials in the Cores of Clusters of Galaxies, in Clusters, Lensing, and the Future of the Universe, eds. V. Trimble and A. Reisenegger, Astronomical Society of the Pacific Conference Series, Vol. 881996.

15. Biviano, aap 297, p. 610 (May 1995).

16. P. D. Mannheim and D. Kazanas, ApJ 342, 635 (July 1989).

17. F. Walter, E. Brinks, W. J. G. de Blok, F. Bigiel, R. C. Kennicutt, M. D. Thornley and A. Leroy, A. J. 136, p. 2563 (2008). 
18. J. R. et. al., The Astrophysical Journal 792, p. 45 (2014).

19. R. Adler, M. Bazin and M. Schiffer, Introduction to general relativityInternational series in pure and applied physics, International series in pure and applied physics (McGraw-Hill, 1965).

20. J. G. O'Brien, J. Dentico, M. Stulge, B. Stefanski, R. J. Moss and S. Chaykov, Journal of Physics: Conference Series, in Review (2016). 